\section{Automation and Application of $a$ Direct-Current Plasma Emission Spectrometer}

\author{
M. S. Epstein \\ Inorganic Analytical Research Division \\ National Bureau of Standards \\ Gaithersburg, MD 20899 \\ R. E. Jenkins \\ Rice University \\ Houston, TX 77251
}

\section{and}

\section{K. S. Epler and T, C. O'Haver}

\author{
Department of Chemistry \\ University of Maryland \\ College Park, MD 20742
}

The direct-current plasma (DCP) coupled to an echelle spectrometer has been used for over 6 years in our laboratory as one of several independent methods for the certification of Standard Reference Materials (SRMs). The demanding certification process requires maximum performance from an analytical method as well as a good understanding of the method capabilities and limitations. We have made a number of modifications to the conventional DCP spectrometer to improve accuracy, precision, and analysis speed. Figure 1 is a schematic diagram of the entire DCP spectrometric system with all modifications and enhancements. This paper discusses these modifications and their application to a number of analytical problems.

\section{Conversion from Unmodulated to Wavelength- Modulated Detection}

The advantages of wavelength-modulated detection over unmodulated detection schemes have been well-documented in the literature [1-3]. Signal-to-noise ratio (SNR) enhancement in the presence of background emission flicker noise, reduction of baseline drift, nulling of line spectral interferences and rapid background correction are some of the advantages of wavelength-modulated detection. Unmodulated detection is superior when spectral resolution is critical or when the limiting system noise is detector shot-noise. The DCP spectrometer and detection system were modified so that either unmodulated or wavelength-modulated schemes could be employed.

\section{Reduction of Spectrometer Wavelength Drift}

Severe nonlinear drift of emission intensity was a recurrent problem with one of our two DCP/ echelle emission spectrometers. The drift was a result of the extreme sensitivity of the spectrometer to thermal variations and vibration. Two approaches were taken to reduce the spectrometer drift. The first involved thermal regulation of the spectrometer baseplate at a temperature higher than attained during normal operation, and vibrational isolation of the spectrometer. These actions significantly reduced both long- and short-term intensity fluctuations.

Since the major heat source affecting the spectrometer was the plasma, another approach was removal of the plasma from contact with the spectrometer baseplate. The plasma was housed in a separate vented enclosure, and an optical rail with appropriate focusing optics was positioned between the spectrometer and the plasma. This configuration also effectively eliminated wavelength and order drift as major sources of intensity drift.

\section{Automation of Sample Introduction, Data Collection, and Processing}

An enhanced Apple II+ microcomputer (3.5 $\mathrm{MHz} 65 \mathrm{C0} 2$ processor) coupled to an autosampler and controlled by a BASIC program was used to automate the DCP spectrometer operations. Figure 2 illustrates both the hardware and software design of the system. The autosampler was controlled through the three game control annunciator outputs of the Apple II+ and autosampler status was monitored through two game control pushbutton inputs. The three annunciator outputs were converted to five control states needed to program the autosampler by a binary-to-BCD converter. Voltage output from the spectrometer, either from a current-to-voltage converter (unmodulated system) or from a lock-in amplifier (wavelength-modulation system), was converted to digital data by a 12-bit ADC and processed by the BASIC program. Standard and sample weights and dilution factors are entered into the program at the start of an analysis and a report of sample concentrations is generated at the completion. Options to apply drift 
correction or to use a standard addition correction are also included in the software.

\section{Removal of Interfering Species Using Ion-Exchange Chromatography}

The most straightforward approach to eliminate matrix-induced interferences, and the one that requires the least number of compromises, is the complete separation of analyte from interfering species. When chromatographic methods are directly coupled to spectrometric methods for online separations, sensitivity and accuracy may be further enhanced by preconcentration of the analyte on the chromatographic column.

We have applied an anion exchange method using an activated alumina (acid form) column for on-line preconcentration of phosphorus. Activated alumina has been found by several researchers $[4,5]$ to be a useful column packing material for adsorption of oxyanions. The column is quite useful for the determination of phosphorus in acid digests of iron and copper-based alloys, since the direct DCP determination is complicated by iron and copper spectral interferences. Phosphorus is retained on the column while iron and copper are not retained and elute with a water wash of the column. With preconcentration times of 30 minutes, phosphorus detection limits are 20 times better than obtained using continuous aspiration. A detection limit of 10 $\mathrm{ng} / \mathrm{mL}$ is obtained under these conditions, corresponding to a detection limit of $0.1 \mu \mathrm{g} / \mathrm{g}$ in the metal alloy. Results from the determination of phosphorus in several SRMs, as shown in table 1, were in good agreement with certified values or other independent techniques.

Table 1. Determination of phosphorus in standard reference materials using preconcentration on an activated alumina column

\begin{tabular}{|c|c|c|c|}
\hline Sample & $P, \mu \mathrm{g} / \mathrm{g}^{\mathrm{a}}$ & Other values & Method $^{b}$ \\
\hline \multicolumn{4}{|l|}{ SRM 1252} \\
\hline Phosphorized copper & $84 \pm 8$ & $85 \pm 2$ & $\begin{array}{l}\text { Molybdivanadophosphoric } \\
\text { acid method (ASTM E-62) } \\
{[6]}\end{array}$ \\
\hline \multicolumn{4}{|l|}{ SRM 875} \\
\hline Cupro-nickel & $21.4 \pm 1.8$ & $20 \pm 5$ & Certified value \\
\hline \multicolumn{4}{|l|}{ SRM 1767} \\
\hline Low-alloy steel & $37 \pm 1$ & $39 \pm 2$ & $\begin{array}{l}\text { Modified ASTM } 350-84 \\
\text { heteropolymolybdate pro- } \\
\text { cedure [7] }\end{array}$ \\
\hline \multicolumn{4}{|l|}{ SRM 365} \\
\hline \multirow[t]{4}{*}{ Electrolytic iron } & $15 \pm 1$ & $12 \pm 2$ & $\begin{array}{l}\text { Modified ASTM } 350-84 \\
\text { heteropolymolybdate pro- } \\
\text { cedure [7] }\end{array}$ \\
\hline & & $14 \pm 2$ & $\begin{array}{l}\text { Molecular absorption } \\
\text { spectrometry [8] }\end{array}$ \\
\hline & & 12.5 & Indirect ICP method [8] \\
\hline & & $25 \pm 5$ & Certified value \\
\hline
\end{tabular}

\footnotetext{
${ }^{a}$ Uncertainty expressed as $95 \%$ confidence limits.

${ }^{b}$ Values obtained by other techniques or certified values.
} 


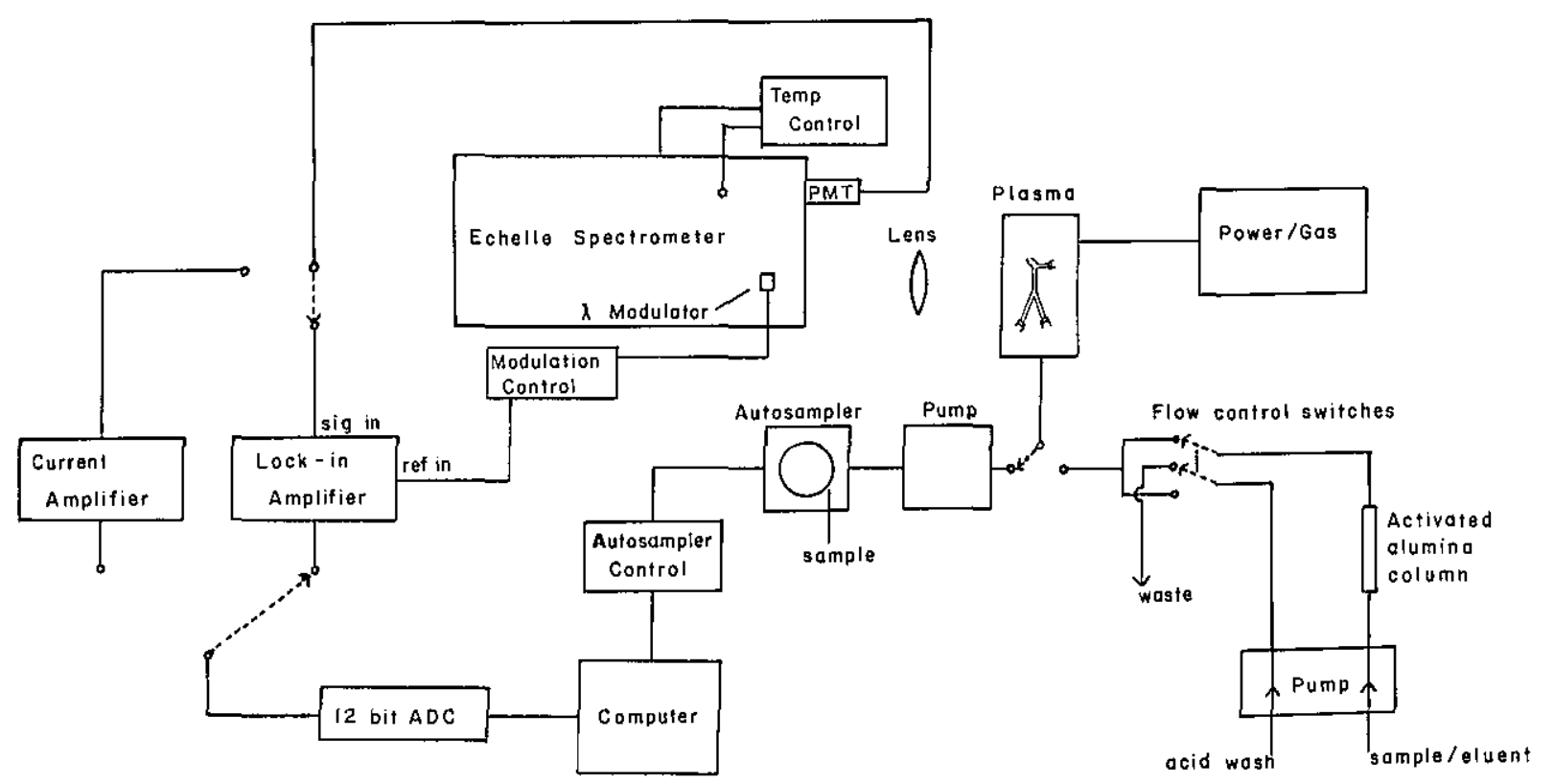

Figure 1. Schematic diagram showing all components of the DCP spectrometric system. 


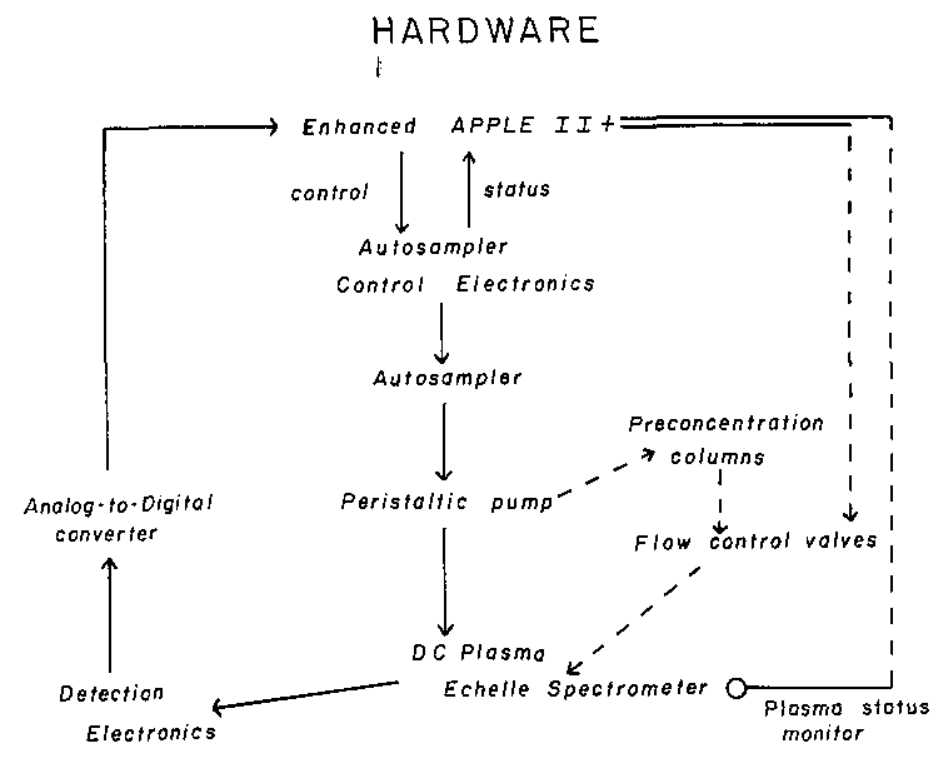

SOF TWARE

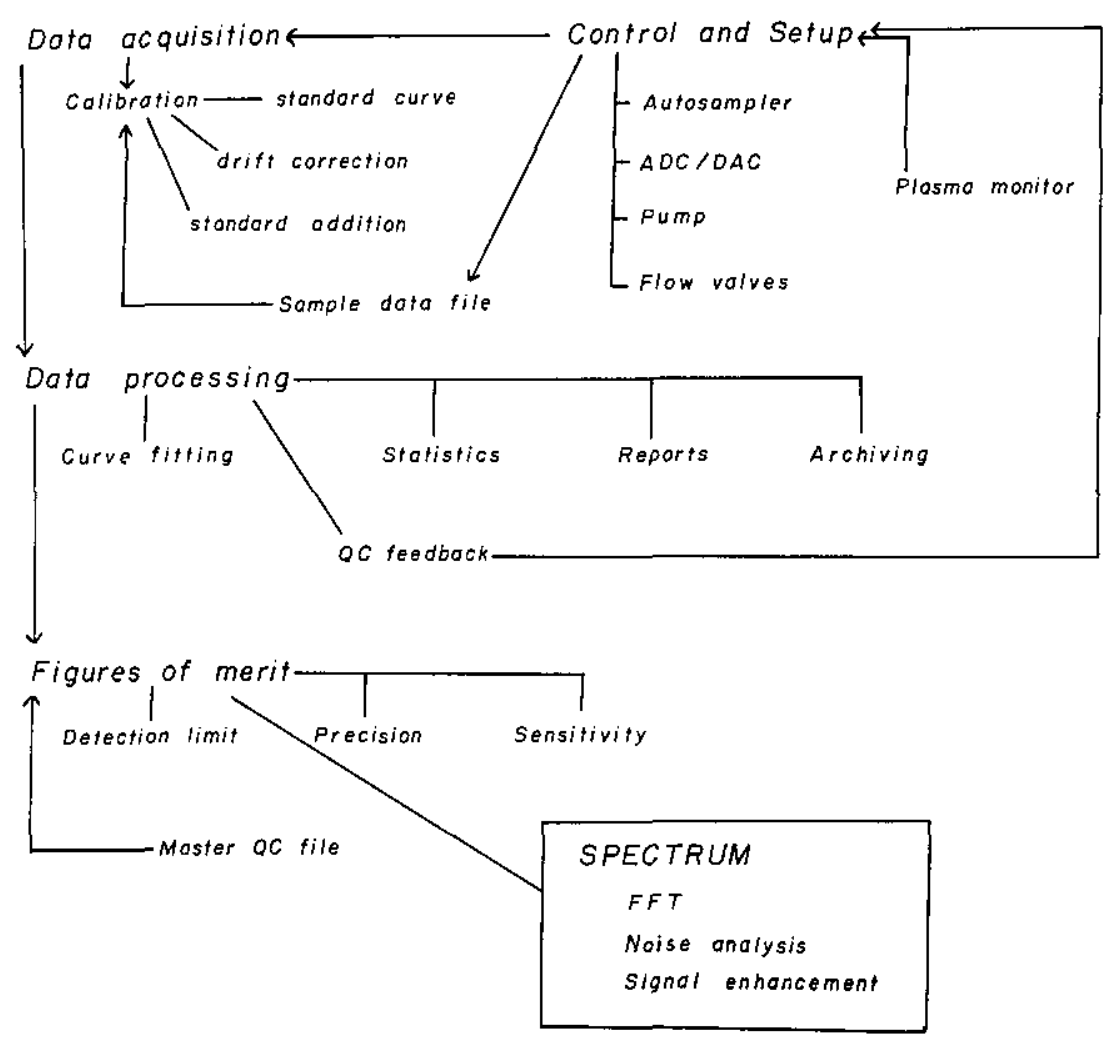

Figure 2. Software and hardware flow diagrams for the DCP spectrometric system. Dashed lines in the hardware diagram indicate components that are not yet automated. 


\section{References}

[1] Snelleman, W., Rains, T. C., Yee, K. W., Cook, H. D., and Menis, O., Anal. Chem. 42, 394 (1970).

[2] Epstein, M. S., and O'Haver, T. C., Spectrochim. Acta 30B, 135 (1975).

[3] Epstein, M. S., and Winefordner, J. D., Prog. Analyt. Atom. Spectrosc. 7, 67 (1984).

[4] McL.eod, C. W., Cook, I. G., Worsfold, P. J., Davies, J. E., and Queay, J., Spectrochim. Acta 40B, 57 (1985).

[5] Cook, I. G., McLeod, C. W., and Worsfold, P. J., Anal. Proc. 23, 5 (1986).

[6] Deardorff, E. R., Private Communication, National Bureau of Standards, March 1981.

[7] Smith, M. V., and Burke, R. W., Private Communication, National Bureau of Standards, August 1987.

[8] Wittman, A., and Schuster, L., Spectrochim. Acta 42B, 413 (1987).

\section{Flow Injection-Inductively Coupled Plasma Spectrometry: $A$ New Strategy for Ultratrace Analysis}

\author{
C. W. McLeod, Y. Zhang, I. Cook, \\ and A. Cox
}

Department of Chemistry

Sheffield City Polytechnic

Sheffield, S1 1WB, U.K.

\section{and}

\author{
A. R. Date and Y. Y. Cheung \\ British Geological Survey \\ 64 Grays Inn Road \\ London, WC1X 8NG, U.K.
}

In the last decade few analytical techniques have made a greater impact in trace inorganic analysis than ICP emission spectrometry. Noteworthy features of the technique are well documented, but lack of sensitivity and susceptibility to spectral interferences have ensured that alternative techniques such as atomic absorption spectrometry remain competitive. The conventional approach to improving method sensitivity is to undertake relatively time-consuming sample pretreatment procedures such as ion exchange, solvent extraction, co-precipitation etc. With the advent of flow injection analysis [1] there has been a considerable interest in the development of on-line microcolumn concentration techniques for atomic spectrometry
$[2,3]$. In the experiment relatively large volumes of sample are passed through a microcolumn in the flow injection (FI) manifold and retained analytes are subsequently eluted by injection of a small volume of eluent. Activated alumina offers a novel route for analyte preconcentration since it can function both as an anion and a cation exchanger depending upon solution $\mathrm{pH}$. Under acidic and basic conditions alumina exhibits a high affinity for oxyanions and cations, respectively:

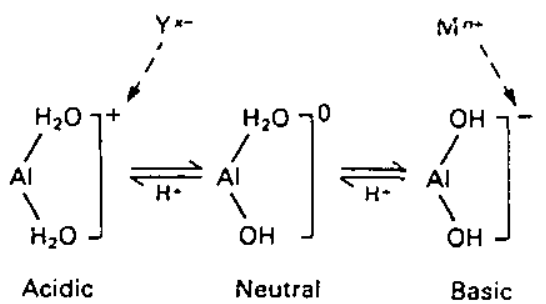

\section{On-line Trace Enrichment: Acidic Alumina}

The acidic form of alumina has a high affinity for a range of oxyanions and, as a result, FI-ICP methodology has been devised for the determination of phosphorus in steels, chromium VI in waters and sulphate in waters. The FI manifold consisted of a peristaltic pump, dual injection rotary valve, and a microcolumn $(20 \mathrm{~mm} \times 1.5 \mathrm{~mm}$ i.d.) of acidic alumina. In operation, samples were injected into the carrier stream and oxyanions were retained on the column whereas cationic species were unretained. An injection of ammonium or potassium hydroxide was then used to elute analyte into the ICP. Typical emission-time responses corresponding to on-line enrichment of chromate are presented in figure 1. The approach provided the basis for development of a rapid speciation scheme for inorganic chromium by virtue of the fact that the microcolumn has a high affinity for anionic chromium VI in contrast to that for chromium III. Thus on injection of samples containing the two chromium species, chromium III was not retained by the column and hence time-resolved emission signals corresponding to the two oxidation states were monitored. Analytical data for the determination of chromium III and chromium VI in a reference water (SRM 1643a, National Bureau of Standards) certified for total chromium content are given in table 1 . 